\title{
ESTUDO DA ADSORÇÃO DE BROMETO DE ETÍDEO EM RESINA XAD-7
}

\author{
Mônica Winkler de Oliveira, Alexandre W. S. Hilsdorf, Astréa F. de Souza Silva e André Fernando Oliveira* \\ Núcleo de Ciências Ambientais, Universidade de Mogi das Cruzes, Av. Cândido X. A. Souza, 200 08780-911 Mogi das Cruzes \\ - SP, Brasil
}

Recebido em 3/4/08; aceito em 16/12/08; publicado na web em 11/5/09

\begin{abstract}
STUDY OF ETHIDIUM BROMIDE ADSORPTION ON XAD-7 RESIN. The adsorption of ethidium bromide on XAD-7 resin was studied. The Freundlich model was the most representative isotherm model to describe the sorption behavior. A solid-liquid equilibrium model was proposed to explain the resin mass influence on the sorption. The equilibrium constant value estimated was 2.31. The results showed an ethidium bromide ion-pair physical adsorption, with adsorption enthalpy equals to $-19.33 \mathrm{~kJ} /$ mol. A pK 2 value equals to $4.69 \pm 0.01$ was estimated by two distinct methods. The results will be applied to the ethidium bromide preconcentration aiming its decomposition.
\end{abstract}

Keywords: ethidium bromide; XAD-7 resin; adsorption isotherm.

\section{INTRODUÇÃO}

A Química Verde, um conceito importante atualmente, tem como preocupação o desenvolvimento de tecnologias e processos que levam em consideração os impactos à saúde humana e ao meio ambiente, por meio, por exemplo, da diminuição do uso e da geração de materiais perigosos, gerados pelos inúmeros produtos fundamentais à humanidade. ${ }^{1}$

O brometo de etídeo (EtBr) é um composto derivado da fenantridina, ${ }^{2}$ cujos equilíbrios ácido-base de Bronsted são apresentados na Figura 1, e muito utilizado em laboratórios de biologia molecular para corar ácidos nucléicos, pois, quando intercalado na estrutura de DNA, o sistema formado fluoresce com uma cor vermelhoalaranjado ${ }^{3}$ sob luz ultravioleta. ${ }^{3,4}$ Desta maneira, este composto é um severo poluente devido à capacidade de se intercalar na estrutura do DNA, apresentando um elevado potencial mutagênico e carcinogênico. ${ }^{3-5}$

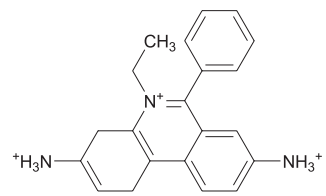

(aq)

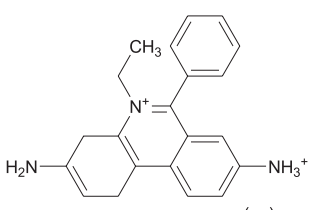

(aq)
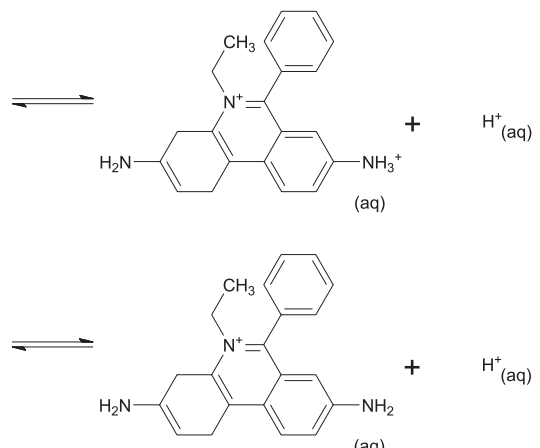

(aq)

Figura 1. Equilíbrios ácido-base de Bronsted-Lowry do sistema Etídeo (CAS 1239-45-8)

Vários procedimentos são descritos para a destruição do brometo de etídeo, principalmente sua adsorção em carvão ativo seguida de incineração do conjunto, ${ }^{6,7}$ enquanto que outros procedimentos incluem a destruição com agentes oxidantes e redutores, ${ }^{2,4}$ como permanganato de potássio e hipoclorito de sódio. Alguns autores,

*e-mail: ferqa@umc.br entretanto, não recomendam essa prática, pois há indícios da produção de resíduos também tóxicos. ${ }^{4}$ Além disso, as baixas concentrações do brometo de etídeo nos resíduos também dificultam sua destruição. ${ }^{2}$

A XAD-7 é uma resina polimérica adsorvente composta por monômeros de éster-acrílico que possui polaridade intermediária. Tem sido usada para remover poluentes orgânicos de resíduos aquosos, para adsorver tanto materiais hidrofóbicos de água como materiais hidrofílicos de sistemas não-aquosos, ${ }^{8}$ assim como na préconcentração de substâncias húmicas ${ }^{9}$ e metais, após adsorção de compostos quelantes..$^{10}$

O estudo do comportamento do EtBr sobre XAD-7 é importante para o desenvolvimento de metodologia para pré-concentração desse composto, visando posterior destruição. Espera-se a adsorção deste cátion nesta resina neutra, através da formação de par iônico. ${ }^{11} \mathrm{O}$ objetivo deste trabalho foi, portanto, estudar o processo de adsorção do brometo de etídeo em resina XAD-7.

\section{PARTE EXPERIMENTAL}

Todos os reagentes utilizados foram de grau analítico e usou-se água deionizada em todos os experimentos. Os espectros de absorção foram obtidos em espectrofotômetro UV-visível Agilent 8453A com cubetas de quartzo com caminho óptico de $10 \mathrm{~mm}$.

Solução de brometo de etídeo - (EtBr) (Merck) 1,23.10-3 mol/L. As demais soluções de trabalho foram preparadas a partir dessa solução estoque por diluição.

Solução tampão universal - foi preparada uma solução contendo citrato $0,05 \mathrm{~mol} / \mathrm{L}$, fosfato $0,04 \mathrm{~mol} / \mathrm{L}$ e ácido bórico $0,05 \mathrm{~mol} / \mathrm{L}$, cujo $\mathrm{pH}$ foi ajustado com $\mathrm{HCl}$ 4,8 mol/L. A força iônica foi corrigida com $\mathrm{NaCl}$ para o valor de $0,5 \mathrm{~mol} / \mathrm{L}$.

Pré-condicionamento da resina XAD-7 - para garantir a ausência de substâncias previamente adsorvidas à resina, uma massa de resina nova foi lavada com água deionizada e mantida em uma solução de $\mathrm{HCl}$ 0,12 mol/L, sob agitação magnética por $4 \mathrm{~h}$. A solução foi filtrada e após a lavagem da resina, com água deionizada, foi mantida em uma solução de $\mathrm{NaOH}$ 0,1 mo/L (Synth) sob agitação magnética por mais $4 \mathrm{~h}$. Após este tempo, a resina foi novamente lavada e mantida em água deionizada por uma noite. $\mathrm{O}$ tratamento foi finalizado mantendo-se a resina em metanol (J.T Baker) sob agitação por $4 \mathrm{~h}$ e depois de filtrada, seca em dessecador a vácuo. ${ }^{12}$ 


\section{Procedimento}

Para obtenção das isotermas utilizou-se $10 \mathrm{~mL}$ de cada solução de $\mathrm{EtBr}$ com concentrações de 10 a $100 \mu \mathrm{mol} / \mathrm{L}$, em béqueres contendo diferentes massas de resina de 20 a $500 \mathrm{mg}$. As misturas foram mantidas em agitação magnética por 10 min e a concentração de $\mathrm{EtBr}$ em equilíbrio foi determinada no sobrenadante.

Os efeitos do $\mathrm{pH}$ e da temperatura na adsorção de soluto foram avaliados com variação no $\mathrm{pH}$ de 2,0 a 7,0 e da temperatura de 10 a $40{ }^{\circ} \mathrm{C}$, utilizando-se uma massa constante de $200 \mathrm{mg}$ de resina.

A resina também foi avaliada em termos de retenção de prótons. Para este estudo, uma coluna contendo a resina foi percolada com 2 $\mathrm{mL}$ de ácido clorídrico $1 \mathrm{~mol} / \mathrm{L}$ e lavada com água. Posteriormente pesou-se $500 \mathrm{mg}$ dessa resina, colocou-se $5 \mathrm{~mL}$ de água e a mistura foi titulada com hidróxido de sódio 0,09 mol/L.

\section{RESULTADOS E DISCUSSÃO}

\section{Caracterização e método de quantificação do brometo de etídeo}

Para a determinação da concentração de brometo de etídeo na fase aquosa, optou-se pela espectrofotometria molecular. O espectro de absorção do composto apresenta 4 máximos de absorção em 213, 286,317 e $480 \mathrm{~nm}$.

Os valores de absortividades molares aparentes referentes aos comprimentos de onda citados foram determinados mediante curvas analíticas obtendo-se de 4,4.10 $; 5,5.10^{4} ; 1,6.10^{4}$ e $5,9.10^{3} \mathrm{~L} /(\mathrm{mol} \mathrm{cm})$, respectivamente, para 213, 286, 317 e $480 \mathrm{~nm}$. Desta maneira, foi escolhido o comprimento de onda igual a $286 \mathrm{~nm}$ para o monitoramento da concentração de brometo de etídeo por apresentar a maior sensibilidade da curva analítica (Figura 2).

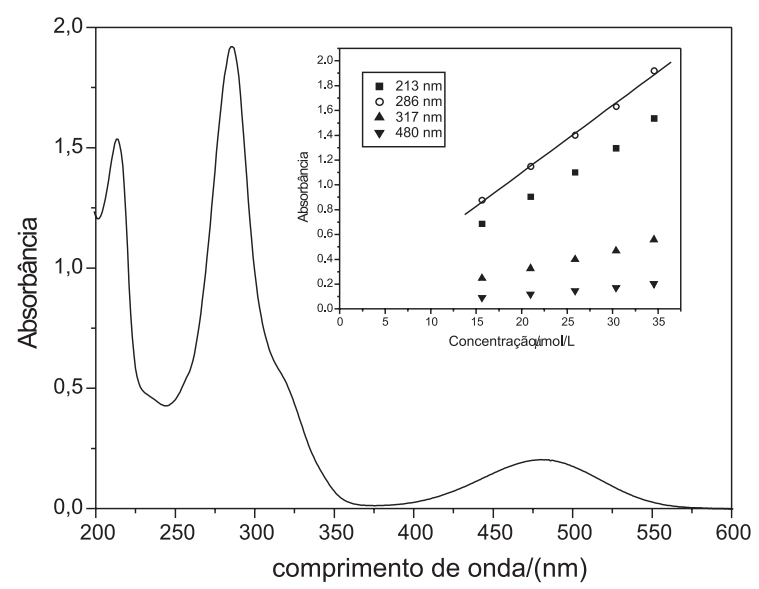

Figura 2. Espectro de absorção molecular de solução de EtBr 35 mol/L. No detalhe, são apresentadas as curvas analíticas nos valores de comprimentos de onda com máximo de absorção

O comportamento do brometo de etídeo foi estudado em diversos valores de $\mathrm{pH}$ em tampão universal, conforme apresentado na Figura 3 , a fim de compreender o seu comportamento ácido-base. Com o aumento do $\mathrm{pH}$ do meio, observa-se o aumento da banda na região de $213 \mathrm{~nm}$ e a diminuição em $226 \mathrm{~nm}$ devido à diminuição da concentração da espécie protonada. A elevação da linha base abaixo de $213 \mathrm{~nm}$ foi devida à presença do tampão universal.

A influência do pH no espectro é devido ao equilíbrio de transferência de prótons (equilíbrio ácido-base de Bronsted-Lowry). Pela análise da estrutura da molécula, é possível estimar dois sítios

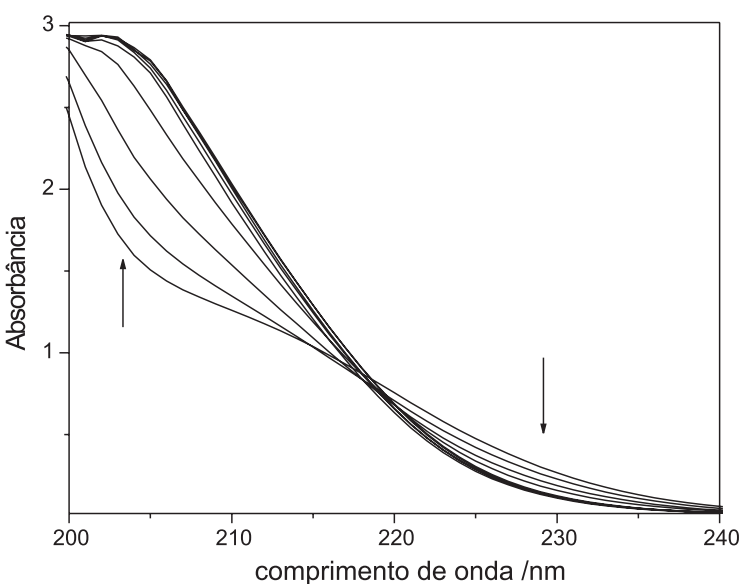

Figura 3. Espectros de EtBr 0,15 $\mu \mathrm{mol} / \mathrm{L}$ em diferentes valores de $\mathrm{pH}$. Setas indicam valores crescentes de $\mathrm{pH}$

de protonação, ou seja, as aminas aromáticas, as quais apresentam, usualmente, valores de pKa menores que 5,0, mas, devido à conjugação na fenantridina, uma delas devem apresentar um valor algumas unidades mais baixo. ${ }^{13}$

Assim, os graus de dissociação foram então estimados, considerando-se um sistema pseudo-monoprótico e, portanto, utilizando-se a Equação $1^{14}$

$\alpha_{i}=\frac{A_{j}-B}{A_{\text {máx }}-B}$

onde $\alpha_{i}$ é o grau de dissociação para a espécie a espécie 'i'; $\mathrm{A}_{\mathrm{j}}$ é a absorbância no comprimento de onda (205 e $226 \mathrm{~nm}$ ) para o pH igual a j; $A_{\max }$, a maior absorbância medida nesse comprimento de onda no $\mathrm{pH}$ onde se supõe que o grau de dissociação seja igual a 1 e, B é o valor do branco nesse mesmo comprimento de onda.

A partir dos dados experimentais, onde é observado apenas um equilíbrio ácido-base, considera-se que o próton removido nessa faixa de $\mathrm{pH}$ corresponde ao segundo próton ionizável do composto (associado ao segundo pKa do composto), devido à elevada acidez do primeiro sítio de protonação, como já discutido.

Desta maneira o $\mathrm{pK}_{2}$ foi estimado por dois métodos distintos (Figura 4). O primeiro, utilizando a forma logarítmica da equação da constante de acidez (Equação 2)

$p H=p K_{2}+\log \left(\frac{\alpha_{2}}{\alpha_{1}}\right)$

onde $\alpha_{1}$ e $\alpha_{2}$ são, respectivamente, os graus de dissociação da espécie protonada e com um próton a menos. Foi verificado o comportamento linear da Equação 2 sendo, então, o pKa estimado de 4,69 \pm 0,01 estimado por regressão linear (Figura 4).

Para a estimativa do pKa foi também testado um método nãolinear sigmoidal (sigmóide de Boltzmann) para a curva de $\alpha_{2}$ em função do pH (Figura 4). Este método é útil para determinar o pKa em sistemas cuja resposta analítica (R) é função apenas de uma espécie do equilíbrio ácido - base pela Equação 3

$\mathrm{R}(\mathrm{pH})=\mathrm{R}_{\mathrm{i}} \cdot \alpha_{\mathrm{i}}+\mathrm{R}_{\mathrm{o}}$,

onde $\mathrm{R}_{\mathrm{o}}$ é uma componente constante da resposta que não é influenciada pelo $\mathrm{pH}$ e $\mathrm{R}_{\mathrm{i}}$ é a resposta da espécie que influencia o sistema. Assim, considerando-se um sistema pseudomonopróticos, o grau de dissociação pode ser calculado pela Equação 4. 

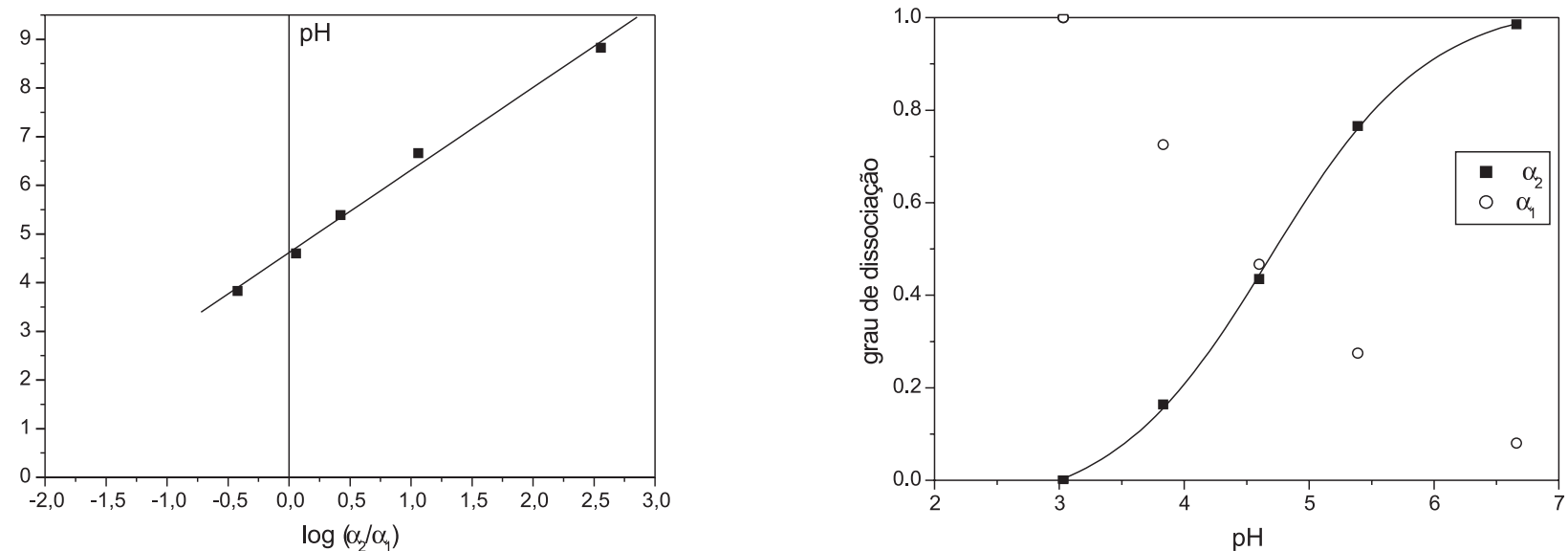

Figura 4. Estimativa do $\mathrm{pK}_{2}$ do EtBr por dois métodos: (A) método de linearização; (B) método não-linear sigmoidal

$\alpha_{2}=\frac{1}{\left(1+10^{(p H-p K a)}\right)}$

Assim, foram obtidos os parâmetros para o modelo não-linear apresentado na Equação 5, com auxílio do software Microcal Origin 6.0

$y=\frac{\left(A_{1}-A_{2}\right)}{\left(1+e^{\left(x-x_{o}\right) / d x}\right)}+A_{2}$

onde os termos $\mathrm{A}_{1}$ e $\mathrm{A}_{2}$ devem diferir por uma unidade, sendo o segundo igual à absorbância da solução na condição em que o grau de dissociação do composto é igual a 0 , ou seja, o valor do branco. $\mathrm{O}$ valor estimado do expoente $\mathrm{x}_{\mathrm{o}}$ é igual ao $\mathrm{pKa}$, fixando-se o termo $\mathrm{dx}$ igual a $1 / \ln (10)$, ou seja, 0,43429 . O valor de pKa obtido foi igual a $4,69 \pm 0,05$. Este último método é mais interessante, pois não exige que a resposta seja obtida em um grande intervalo de $\mathrm{pH}$.

A importância da determinação dos valores de pKa de um sistema químico em qualquer estudo em solução (principalmente em solução aquosa) é a compreensão do comportamento das espécies desse sistema em solução.

\section{Estudos de adsorção}

No estudo das isotermas do brometo de etídeo adsorvido em resina XAD-7, utilizaram-se diferentes massas da resina e o valor do $\mathrm{pH}$ das soluções foi mantido em 7,0, valor em que as aminas estão desprotonadas. A partir das medidas de absorbância (em $286 \mathrm{~nm}$ ), realizadas após o tempo de agitação e repouso, foram calculadas as quantidades de brometo de etídeo adsorvidas. É importante ressaltar que os valores de $\mathrm{pH}$ das soluções foram sempre ajustados para o mesmo valor antes das medidas de absorbância (isto é, igual 4,0) para garantir o mesmo comportamento do sistema em relação à curva analítica.

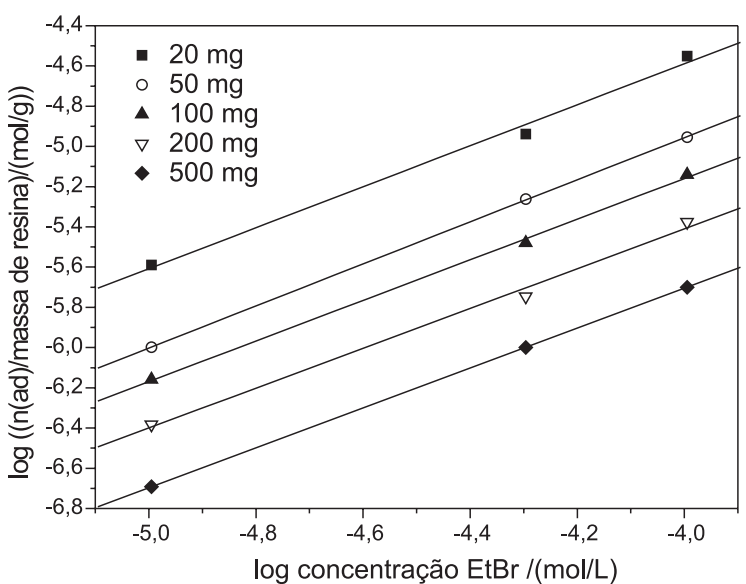

Figura 5. Isotermas de Freundlich para EtBr em diferentes massas de resina $X A D-7$

O modelo de Langmuir não se mostrou adequado para os resultados obtidos. Foi estudado o modelo da isoterma de Freundlich (Equação 6) o qual apresentou um comportamento linear em todas as condições estudadas, como pode ser observado na Figura 5.

$\left[E t B r_{s}\right]=K_{f} \cdot C_{T}^{1 / b}$

onde $\mathrm{K}_{\mathrm{f}}$ é capacidade de adsorção e 1/b é intensidade de adsorção. Esses parâmetros foram obtidos por regressão linear da curva de linearização (Equação 7) e são apresentados na Tabela 1.

$\log \left[E t B r_{s}\right]=\log K_{f}+\frac{1}{b} \log C_{T}$

Tabela 1. Parâmetros obtidos para isoterma de Freundlich, em pH 4,0, a partir da Equação 7

\begin{tabular}{lcccc}
\hline Massa de resina/mg & Kf & $1 / n$ & $\mathrm{r}^{2}$ & sd \\
\hline 20 & $0,31 \pm 0,29$ & $1,02 \pm 0,08$ & 0,9934 & 0,0601 \\
50 & $0,17 \pm 0,01$ & $1,00 \pm 0,70$ & 1,0000 & 0,0053 \\
100 & $0,076 \pm 0,028$ & $1,01 \pm 0,04$ & 0,9988 & 0,0254 \\
200 & $0,035 \pm 0,029$ & $0,99 \pm 0,07$ & 0,9945 & 0,0533 \\
500 & $0,018 \pm 0,000$ & $0,9920 \pm 0,0002$ & 1,0000 & 0,0001 \\
\hline
\end{tabular}

Kf, capacidade de adsorção; 1/n intensidade de absorção, $\mathrm{r}^{2}$ coeficiente de determinação e sd, estimativa dos desvios padrão dos resíduos 
Assim, a inclinação (1/b) não diferiu significativamente da unidade, ao nível de $95 \%$ de confiança ${ }^{15}$ para todas as condições experimentais estudadas. Desta maneira, a isoterma de Freundlich é chamada "linear", ${ }^{16}$ ou tipo constant partition. ${ }^{17}$

$\mathrm{O}$ coeficiente de adsorção Kf permite identificar a capacidade do adsorvente em reter um soluto; pode ser entendido como uma medida da distribuição de equilíbrio entre as fases líquida e sólida. ${ }^{18}$ Os valores apresentados na Tabela 1 sugerem interações de natureza dispersiva (forças de London). ${ }^{19}$

Ainda que o modelo de Freundlich seja usualmente tratado como empírico, para racionalizar os resultados tomou-se por base um modelo de equilíbrio de adsorção sólido-líquido, considerando-se o par-iônico do brometo de etídeo

$$
\operatorname{EtBr}_{(\mathrm{aq})} \rightleftharpoons \mathrm{EtBr}_{(\mathrm{s})}
$$

quantitativamente definido pela constante de equilíbrio $\mathrm{K}_{\mathrm{s}}$ e pelo balanço de matéria,

$$
\begin{aligned}
& K_{s}=\frac{\left[E t B r_{(s)}\right]}{\left[E t B r_{(a q)}\right]} \\
& n_{T}=n_{R}+n_{a q}=C_{T} V_{a q}=\left[E t B r_{(s)}\right] \cdot m_{R}+\left[E t B r_{(a q)}\right] \cdot V_{a q}
\end{aligned}
$$

onde $\mathrm{n}$ é a quantidade de matéria de brometo de etídeo, os sub-índices $\mathrm{R}$ e aq se referem às fases resina e aquosa, respectivamente. $\mathrm{C}_{\mathrm{T}}$ é a concentração total de brometo de etídeo inicialmente na fase aquosa antes de estar em contato com a resina isenta desse composto.

As Equações 9 e 10 podem ser rearranjadas para estabelecer a relação entre a concentração de brometo de etídeo adsorvido e a sua concentração total no sistema.

$C_{T}=\left[E t B r_{(s)}\right] \cdot\left(\frac{m_{R}}{V_{o}}+\frac{1}{K_{s}}\right) \Rightarrow\left[E t B r_{(s)}\right]=\frac{C_{T}}{\left(\frac{m_{R}}{V_{o}}+\frac{1}{K_{s}}\right)}=K_{f} C_{T}$

Assim, a Equação 11 é uma expressão clássica da Equação de Freundlich linear, se $\mathrm{pH}$, temperatura e força iônica são mantidos constantes. É interessante observar que o modelo utilizado é análogo ao equilíbrio de partição. ${ }^{20}$

A relação do termo linear com a massa de resina pode ser obtida a partir da Equação 7.

$$
\frac{1}{K_{f}}=\left(\frac{1}{V_{o}}\right) m_{R}+\left(\frac{1}{K_{s}}\right)
$$

Na Figura 6 é apresentado o gráfico da recíproca dos termos lineares da Equação logarítmica de Freundlich e a massa de resina, com valores apresentados na Tabela 1. Observando o comportamento

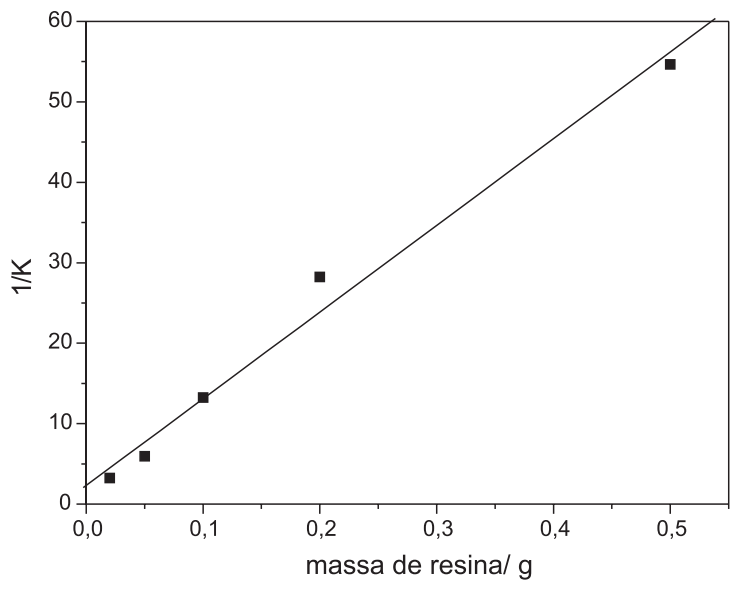

Figura 6. Relação da capacidade de absorção $K_{f}$ e massa da resina

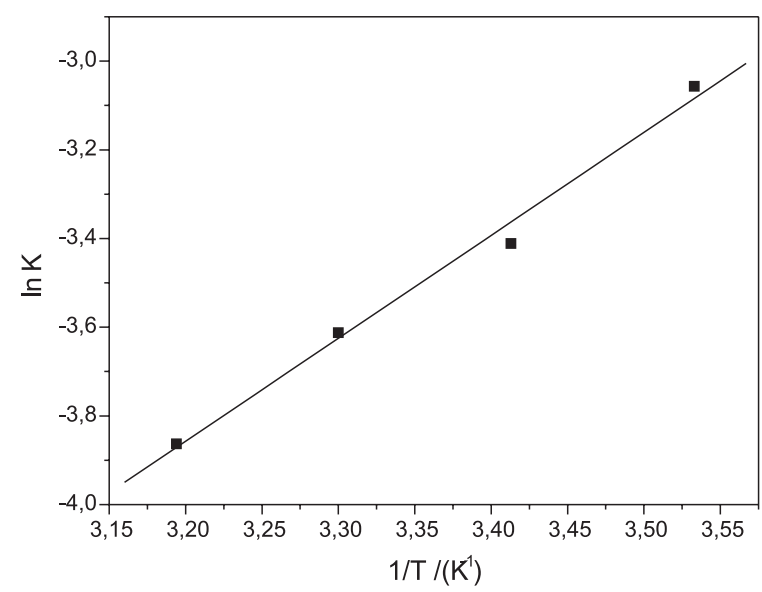

Figura 7. Estimativa da entalpia de adsorção mediante a Equação de van't Hoff

linear previsto na Equação 12, estimou-se a constante de equilíbrio de adsorção condicional $\mathrm{K}_{\mathrm{s}}$ em $2,31 \mathrm{~L} / \mathrm{mg}$ a $25^{\circ} \mathrm{C}$.

As isotermas de Freundlich em diferentes temperaturas foram estudadas e a entalpia de adsorção foi obtida mediante o gráfico de ln $\mathrm{K}_{\mathrm{f}}$ versus 1/T (Figura 7) e através da Equação de van’t Hoff (Equação 13). Utilizando-se dos valores de $\mathrm{K}_{\mathrm{f}}$ obtidos (Tabela 2), estimou-se a entalpia de adsorção em $-19,7 \pm 1,1 \mathrm{~kJ} / \mathrm{mol}$. Isto significa que a adsorção é um processo exotérmico, ou seja, o valor de $\mathrm{K}_{\mathrm{f}}$ tende a diminuir à medida que a temperatura se eleva. ${ }^{21}$ Além disso, esse valor é compatível com aqueles processos relacionados a uma adsorção física. ${ }^{22}$

$\frac{d\left(\ln K_{f}\right)}{d(1 / T)}=-\frac{\Delta H_{a d}}{R}$

A fim de avaliar a presença de grupos carboxílicos residuais na resina devido à eventual hidrólise dos ésteres acrílicos, a qual

Tabela 2. Resultados do ajuste das isotermas de Freundlich com o aumento de temperatura

\begin{tabular}{lcccc}
\hline $\mathrm{T} /\left({ }^{\circ} \mathrm{C}\right)$ & $\mathrm{K}_{\mathrm{f}} / \mathrm{K}$ & $1 / \mathrm{n}$ & $\mathrm{r}^{2}$ & $\mathrm{sd}$ \\
\hline 10 & $0,047 \pm 0,015$ & $1,024 \pm 0,016$ & 0,9998 & 0,0112 \\
20 & $0,033 \pm 0,041$ & $0,9864 \pm 0,0572$ & 0,9967 & 0,0415 \\
30 & $0,027 \pm 0,062$ & $0,9663 \pm 0,0950$ & 0,9904 & 0,0689 \\
40 & $0,021 \pm 0,007$ & $0,9427 \pm 0,0158$ & 0,9997 & 0,0113 \\
\hline
\end{tabular}


poderia então atuar como uma resina aniônica fraca, uma massa de resina foi titulada com $\mathrm{NaOH}$ após ter sido eluída com $\mathrm{HCl}$ 1,0 mL e lavada com água. Não foram observados nenhuma desprotonação nem ponto de equivalência, de maneira que é razoável considerar a resina realmente neutra.

No estudo de adsorção em soluções com diferentes valores de $\mathrm{pH}$, não foram observadas variações significativas na adsorção, o que poderia ser atribuído ao fato de não haver a formação de cargas na resina ou no composto e a adsorção ser mesmo de natureza física, onde o adsorbato se encontra ligado à superfície por forças de van der Waals. ${ }^{22}$ Com essas evidências, sugere-se que esteja havendo a adsorção do par-iônico brometo de etídeo e não apenas do cátion. A estabilidade do par iônico está relacionada à grande polarizabilidade do ânion e ao tamanho do cátion. ${ }^{23}$

\section{CONCLUSÕES}

O comportamento do brometo de etídeo em solução foi caracterizado com auxílio da determinação do $\mathrm{pK}_{2}$ utilizando-se dois métodos distintos para esse cálculo. $\mathrm{O}$ valor obtido foi concordante entre ambos e igual a 4,69 $\pm 0,01$. A isoterma linear de Freundlich mostrou-se adequada para explicar a adsorção de EtBr sobre a resina XAD-7 e um modelo de equilíbrio sólido-líquido foi aplicado para esclarecer a influência da massa de resina sobre a adsorção. A constante de equilíbrio envolvida foi também estimada e com um valor igual a 2,31. O pH da solução não influenciou significativamente o sistema, enquanto que com o aumento da temperatura se determinou a entalpia de adsorção da ordem de -19,33 $\mathrm{kJ} / \mathrm{mol}$, que indica adsorções por interações físicas apenas.

\section{REFERÊNCIAS}

1. Prado, A. G. S.; Quim. Nova 2003, 26, 738.

2. Zocher, R.; Billich, A.; Keller, U.; Mesnner, P.; Biol. Chem. 1988, 369, 1191.

3. http://en.wikipedia.org/wiki/Ethidium_bromide, acessada em Março 2008.

4. Lunn, G.; Sansone, E. B.; Anal. Biochem. 1987, 162, 453.

5. Quillardet, P.; Hofnung, M. O.; Trends Genet. 1988, 4, 89.

6. Olivier, B.; Trends Genet. 1988, 4, 90.

7. Hengen, P. N.; TIBS 1994, 19, 257.
8. http://www.sigmaaldrich.com/chemistry/aldrich-chemistry/techbulletins/al-142/amberlite-amberlyst.html, acessada em Dezembro 2008; http://www.sigmaaldrich.com/catalog/ProductDetail. do?N4=XAD7|SIGMA\&N5=SEARCH_CONCAT_PNO|BRAND_ $\mathrm{KEY} \& \mathrm{~F}=\mathrm{SPEC}$, acessada em Dezembro 2008.

9. Santos, A.; Botero, W. G.; Bellin, I. C.; Oliveira, L. C.; Rocha, J. C.; Mendonça, A. G. R.; Godinho, A. F.; J. Braz. Chem. Soc. 2007, 18, 824.; Rocha, J. C.; Rosa, A. H.; Substâncias húmicas aquáticas Interações com espécies metálicas, $1^{\mathrm{a}}$ ed., Ed. da UNESP: São Paulo, 2003.

10. Tewari, P. K.; Singh, A. K.; Fresenius J. Anal. Chem. 2000, 367, 562; Subramanian, K. S.; Meranger, J. C.; Wan, C. C.; Corsini, A.; Int. J. Environ. Anal. Chem. 1985, 19, 261.

11. Yang, W.; Xia, M.; Li, A.; Yang, L.; Zhan, Q.; React. Funct. Polym. 2007, 67, 609; Casado-Torrones, S.; Fernández-Sánchez, J. F.; Díaz, B. C.; Carretero, A. S.; Fernández-Gutiérrez, A.; J. Pharm. Biom. Anal. 2005, 38, 785 .

12. Santos, H.O.; Dissertação de Mestrado, Universidade de Mogi das Cruzes, Brasil, 2006.

13. Sykes, P.; A Guidebook to Mechanism in Organic Chemistry, $6^{\text {th }}$ ed., Pearson: Malásia, 1986; Oliveira, A. F.; Equilíbrio em solução aquosa orientado à aplicação, Átomo: Campinas, 2009, no prelo; Clayden, J.; Greeves, N.; Warren, S.; Wothers, P.; Organic Chemistry, Oxford University: Oxford, 2001.

14. Previdello, B. A. F.; Carvalho, F. R.; Tessaro, A. L.; Souza, V. R.; Hioka, N.; Quim. Nova 2006, 29, 600.

15. Massart, D. L.; Vandeginste, B. G.; Deming, M. S.; Michotte, N. Y.; Kaufman, L.; Chemometrics: a Textbook, Elsevier: Amsterdam, 1988.

16. Wedler, G.; Manual de Química Física, Calouste Gulbenkian: Lisboa, 2001.

17. Falone, S. Z.; Vieira, E. M. ; Quim. Nova 2004, 27, 849.

18. Alcântara, M. A. K.; Camargo, O. A.: Sci. Agri. 2001, 58, 567.

19. Guilarduci, V. V. S.; Mesquita, J. P.; Martelli, P. B.; Gorgulho, H. F.; Quim. Nova 2006, 29, 1226.

20. Stumm, W.; Morgan, J. J.; Aquatic Chemistry, $3^{\text {rd }}$ ed., Wiley Interscience: New York, 1996.

21. Atkins, P.; Físico-química, LTC: Rio de Janeiro, 2002, vol. 1.

22. Shaw, D. J.; Introdução à química dos colóides e de superfícies, EDUSP/ Edgard Blucher: São Paulo, 1975.

23. Huheey, J. E.; Keiter, E. A.; Keiter, R.; Inorganic Chemistry: Principles of Structure and Reactivity, $4^{\text {th }}$ ed., Prentice Hall: New York, 1977. 